\title{
Evaluation and Exploration of a Social Prescribing Initiative: Study \\ Protocol
}

\author{
Moon M. Halder (moon.halder@ntu.ac.uk), \\ Juliet R. H. Wakefield (juliet.wakefield@ntu.ac.uk), \\ Mhairi Bowe (mhairi.bowe@ntu.ac.uk), \\ Blerina Kellezi (blerina.kellezi@ntu.ac.uk), \\ Elizabeth Mair (elizabeth.mair@ntu.ac.uk), \\ Niamh McNamara (niamh.mcnamara@ntu.ac.uk), \\ Ian Wilson (ian.wilson@ntu.ac.uk) \\ Clifford Stevenson (clifford.stevenson@ntu.ac.uk),
}

Nottingham Trent University, England, UK.

Corresponding author: Clifford Stevenson, Department of Psychology, Nottingham Trent University, 50 Shakespeare Street, Nottingham, NG1 4FQ, UK; clifford.stevenson@ntu.ac.uk 


\begin{abstract}
Attention is being given to healthcare initiatives with the potential to save money and improve lives. One example is Social Prescribing (SP), which supports patients whose illhealth is exacerbated by loneliness. While evidence has accumulated attesting to SP's efficacy, one limitation has been the lack of a theoretical framework, which limits understanding of how tackling loneliness improves health. In our evaluation of an SP pathway, we adopt a 'Social Cure' approach, which posits that social relations affect health. Our study will evaluate the efficacy of the pathway and determine the extent to which group processes are responsible for health improvements.
\end{abstract}




\section{Background}

The Office for National Statistics (2017) predicts that the number of UK inhabitants aged over 85 will double in the next 25 years, leading to an increased need for Health and Social Care. Indeed, it is expected that there will be a social care funding gap of $£ 5.8$ billion by 2020 (Local Government Association, 2017). With this in mind, service-providers have begun to consider adopting healthcare initiatives which have the potential to both save money and improve lives. The initiative explored in this paper involves a radical departure from traditional medical models, where GPs (General Practitioners) are responsible for diagnosing illness and then prescribing appropriate medication. As Brandling and House (2009) note, this approach tends to mean that the psychosocial issues at the root of many chronic health conditions (e.g., depression, type-2 diabetes) are not addressed, leading to such conditions becoming unnecessarily medicalised. If chronic conditions could instead be managed more holistically by enabling patients to make choices that will enhance their overall wellbeing, then this medicalisation (and its associated costs) could be reduced. Wellbeing would be further enhanced if health professionals sought to tackle the key social predictors of ill-health, such as loneliness (Hawkley and Cacioppo, 2010). These principles areThis is at the core of Social Prescribing (SP; e.g., South et al., 2008), which itself underpins the initiative explored in this paper.

\section{What is Social Prescribing?}

SP is a non-clinical approach designed to support individuals experiencing chronic mental/physical health problems exacerbated by loneliness, often leading to increased healthcare appointment attendance (King's Fund, 2017). Although it exists in many forms, SP generally involves providing patients with a range of social, emotional, and practical support (Grant et al., 2000) while also offering them opportunities to take responsibility for their own health. As the name would suggest, SP also has a strong social element: it involves 
health professionals encouraging patients to join voluntary, community, and social enterprise groups within the third-sector (Kimberlee et al., 2014). Common examples include social or lunch clubs, befriending groups, gardening groups, and crafts groups. Patients are supported in their continued membership of these groups. This is facilitated by community or 'Link Workers' (LWs) who use their local community knowledge to connect patients to relevant groups. In this way, SP bridges the gap between primary healthcare and the community sector, thereby providing personal support that is tailored to individuals' needs (South et al., 2008).

SP initiatives have become increasingly common within the UK's National Health Service (NHS), and have shown good evidence of success. For example, SP has helped patients with psychological problems build confidence, develop skills, and express themselves (Stickley and Hui, 2012), as well as experience reductions in anxiety, improved ability to carry out everyday activities, and improved feelings of general health, wellbeing, and quality of life (Grant et al., 2000). Such findings suggest that SP has the potential to promote fundamental change by empowering patients to identify their strengths and skills, thus helping them manage their own health. Return on investment has also been reported (Dayson and Bashir 2014; Kimberlee et al., 2016), suggesting the potential benefits of SP also extend into the financial sphere, and could thus reduce NHS budget overspends without compromising patient care. In light of such evidence, the use of SP alongside usual healthcare has been advocated by GPs (Cawston, 2011).

\section{Applying Social Psychology to Social Prescribing}

While such findings are encouraging, we argue that an important limitation of the SP interventions outlined in previous literature is that they lack a clear theoretical framework. For example, whilst Kimberlee's (2015) review of SP in England involves categorising the 23 included interventions into types of model (i.e., signposting, light, medium, and holistic), 
they are not identified by any specific theoretical models. Similarly, in their review, Thomson et al. (2015) describe the development of SP as being influenced by principles including self$\underline{\text { help, social capital, and social connectivity, but a clear guiding theoretical framework }}$ remains absent. Such a framework is important because it allows researchers and practitioners to understand more about the processes through which SP might promote improvements in patient wellbeing. Attempting to remedy this shortcoming, the current evaluation is based upon a specific theoretical framework: the social identity approach to health and well-being, named 'The Social Cure' (Jetten et al, 2012). This is based upon we have underpinned this evaluation with-the Social Identity Approach (SIA) within social psychology, which. This approach, comprised of the principles of Social Identity Theory (Tajfel and Turner, 1986) and Self-Categorisation Theory (Turner et al., 1987), posits that we are all members of multiple social groups, and that at any one time our membership of a particular group can become salient (conspicuous) to us. This encourages us to adopt the 'contents' of that particular group - its norms, values, and attitudes. This is especially true if we identify strongly with the group, whereby we experience a subjective sense of belonging to the group, and a subjective sense of commonality with its members (Sani et al., 2015).

Over the last decade, various authors conducting research within the SIA have begun to explore the idea that identification with social groups has the potential to benefit one's improve health and wellbeing. Named-Specifically, the 'Social Cure' this approach research (Haslam et al., 2018; Jetten et al., 2012) shows posits that meaningful identification with $\underline{\text { social groups provides individuals with important resources, such as a sense of social }}$

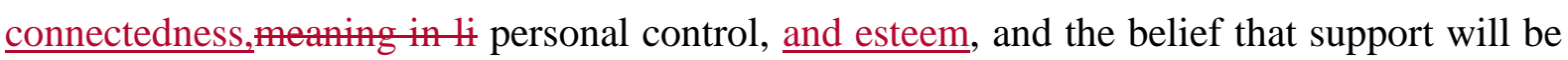
forthcoming from group members during times of crisis. It is through such processes that group identification has been shown to predict improved wellbeing across a range of settings (e.g., Greenaway et al., 2015; Haslam et al., 2005). Indeed, possessing group identifications 
has been shown to predict reductions in depression/anxiety in people with multiple sclerosis (Wakefield et al., 2013), help people deal with extreme life events like war (Kellezi et al., 2009), and allow people to cope better with life transitions, such as starting university (Iyer et al., 2009). The health benefits of group engagement have also been harnessed in a novel group support programme called Groups4Health, recent evaluations of which show sustained improvements in health and wellbeing resulting from successful engagement and identification with the support group (Haslam et al., 2016). In other words, the health benefits of group membership are not incidental to group identification, but demonstrably result from meaningful psychological engagement with supportive others.

We therefore adopt a Social Cure perspective in the present evaluation. Specifically, we intend to not only explore the health-related efficacy of the SP initiative in question, but also to examine the extent to which Social Cure processes help to explain how such healthcare gains occur. By doing this we intend to integrate the as-yet unintegrated domains of SP and Social Cure, which we believe will be of benefit to both literatures. Specifically, we intend to extend the Social Cure literature by exploring a 'real world' application of its principles, and to enrich the SP literature by suggesting a framework though which its undeniable benefits could be more clearly understood.

\section{The SP Pathway}

The SP initiative we are evaluating is a recently-developed NHS-based pathway initiated in 2017 in Nottingham, England. As with many NHS-based SP pathways, the initiative is supplemental to any other healthcare being received by patients, and is intended to improve illness prevention and patient self-management, ultimately resulting in reduced GP attendance. In this particular initiative, leaflets and posters in participating GP surgeries encourage individuals experiencing chronic health conditions and loneliness to refer themselves onto the pathway. In addition, GPs who believe a patient could benefit from SP 
can refer them directly, as can local community organisations. Once identified as being suitable candidates for the initiative, individuals (hereafter service-users) meet with a dedicated health professional (a Health Coach: HC) who assesses their needs, before either prescribing self-care management, or referring them to a community-based Link Worker who can connect them to appropriate third-sector organisations. Service-users are regularly recontacted for monitoring purposes.

\section{Aims}

The aims of this study are two-fold. First, we want to evaluate the efficacy of the pathway by exploring the extent to which the initiative leads to enhanced service-user wellbeing and a resultant reduction in healthcare use. Second, we want to explore the extent to which group processes (namely activity group identification, social support, community integration, and feelings of group resilience or 'collective efficacy') are responsible for these predicted improvements. We make the following predictions:

H1: Those referred to the pathway should evidence a strong relationship between $\underline{\text { social isolation, loneliness, and poorer wellbeing. }}$

H2: Successful referral, assessment and adherence to the pathway should (on aggregate) be associated with improved wellbeing and reduced loneliness.

H3: These improvements will, in part, be mediated by the impact of the programme on the social connectedness of the service-user.

H4 Improvements will likely be moderated by the quality of relationship between the $\underline{\text { service-user and the service-provider }}$

H5: These improvements will be related to a reduced usage of health and social $\underline{\text { services as well as a quantifiable improvement in Quality-Adjusted Life Years. }}$ 
$\underline{\text { In addition, from our qualitative work, we expect to see service-users' experiences of }}$ treatment to relate to their self-reported engagement with their service-providers and activity group members. We also expect to see better service delivery and outcomes when serviceusers and service-providers share a convergent understanding of the treatment purpose.

\section{Methods/Design}

\section{Study Design}

This project involves a survey study and an interview study. We chose to conduct two studies for the purposes of data triangulation (Jick, 1979) as well as allowing us to capture both the longitudinal impact of the pathway and its experiential component.

\section{Study 1: Survey Study}

Study 1 is a five-wave longitudinal quantitative survey which is intended to: i) explore the effect of the initiative on various measures of health and wellbeing (via the evaluation aspect of the study); and ii) to-investigate the potential mediating effect of Social Cure-related variables such as social support and collective efficacy (via the study's research aspect).

\section{Participant Identification and Recruitment}

Anyone can be referred (or self-refer) to the pathway, as long as they are over 18, live in the relevant area in Nottingham (or are registered with a GP in the area), are managing one or more long-term health conditions, and feel isolated, lonely, or anxious. Data will be collected from a specific cohort of-around 400 individuals that join the initiative, with a recruitment target of 400. This will include patients from all of the GP surgeries in the area. People can be recruited into the intervention (and thus potentially into our study) in a number of ways. These include: i) by GPs during consultations; ii) by third-sector organisations operating within Nottingham; and iii) by self-referral after seeing promotional materials in 
GP surgeries, libraries, and community centres. Any referrals will be passed on to pathway staff, who will contact the relevant individuals and ask if they wish to be involved. As part of a broader evaluation of the pathway undertaken by our research team, non-identifying audit level data on the demographic characteristics of all referred individuals can be examined to determine if there are any systematic biases to rates of initial attendance (or participation in the research).

\section{Data Collection}

All survey data will be collected by pathway employees with honorary NHS contracts and data-capture training. Data will be collected and recorded on the NHS SystmOne database.

As mentioned previously, there are two aspects to Study 1: the evaluation survey and the research survey. During the service-user's first ('baseline') meeting with the HC, evaluation survey data will be gathered. Completing the evaluation survey is part of standard care, but service-users will asked if they consent for their anonymised evaluation survey data to be used in our evaluation. Three months later, the service-user will complete the first telephone-based follow-up. This will be repeated three more times, each at three-month intervals. Evaluation survey data will be gathered during each of these conversations. Moreover, for service-users who consent, research survey data will also be gathered during the telephone conversation. Completing the research survey is not part of standard care, but will enable us to explore the Social Cure processes we believe to be at work. While the scales may appear lengthy, the fact that service-users are guided through the surveys in-person/via telephone by a health professional allows them to feel supported and gives them the opportunity to clarify any questions about which they are unsure.

\section{Measures}

\section{Evaluation Survey}


The evaluation survey is designed to establish rapport between the pathway staff and the service-user, and also to establish service-users' healthcare needs, service usage, quality of life, and social activities. The evaluation survey includes the self-report and standardised measures (see Table 1). Unless stated, the mean of each service-user's responses to the multiple items in a single scale is calculated.

Patient activation is measured with the 13-item Patient Activation Measure (PAM), which measures a range of health-related characteristics and behaviours (Hibbard et al., 2005) All items are rated using a 1-4 scale (disagree strongly-agree strongly). Service-users receive a PAM score between 0 and 100, which helps the HC to decide how best to support them.

Weekly activities are recorded by asking service-users to think about how they spend their time over a typical week, with each day divided into morning, afternoon, and evening.

Social group membership information will be recorded by asking service-users to tick the types of social group/s to which they belong. Group-types listed include sports groups, hobby groups, and religious groups. The frequency with which the service-user meets with each group is also recorded (e.g., 'daily', 'weekly', 'monthly' or 'yearly').

Sense of local community belonging is assessed using a single item rated on a 1-4 scale ('definitely not'-'yes definitely'): "Thinking about this local community, the kind of place it is and the kind of people who live around here, would you say that you feel a sense of belonging to this local community?" Service-users are asked to define 'local community' in any way that is meaningful to them. This item has been adapted from previous research (McNamara et al., 2013).

Social support received from others is assessed using a 4-item scale adapted from Haslam et al. (2005). Service-users rate their agreement with each item (e.g., "Do you get the emotional support you need from other people?’) on a 1-5 scale ('not at all'- 'completely'). 
Loneliness-Social isolation is assessed with 8-item scale adapted from Hays and DiMatteo (1987). Service-users rate their agreement with each item (e.g., "I lack companionship") on a 1-5 scale ('not at all'- 'completely').

Quality of life is assessed using the EQ5D scale (EuroQol Group, 1990). Five health dimensions (mobility, self-care, usual activities, pain, and anxiety/depression) are each assessed with a single item on a 1-3 scale (e.g., "I have no problems walking about/I have some problems walking about/I am confined to bed"). Service-users select one option for each dimension.

Health service use is assessed by asking service-users to indicate the number of times that have used five types of service in the last three months (adapted from Kellezi et al., 2016): 'primary care’ (e.g., GP appointments), 'secondary care' (e.g., hospital appointments), 'community care' (e.g., a care-home stay), and 'social care' (e.g., receiving home help).

Finally, sociodemographic data will be recorded at baseline, including the serviceuser's age, gender, housing (e.g., rented, bought, etc.), relationship status, employment status, highest level of education, and which (if any) government benefits they receive.

\section{Research Survey}

The research survey items are intended to explore the group processes we believe to be at work in the initiative, and include self-report and standardised measures (see Table 1). Unless stated, all items are rated on a 1-5 scale (strongly disagree-strongly agree), and the mean of each service-user's responses to the multiple items in a single scale is calculated.

Goal attainment is assessed by asking service-users to list up to 3 goals that they had set during the initial meeting with their $\mathrm{HC}$ and also indicate to which extent they feel they have met each goal on a 1-5 scale (I have not met it at all-I have met it completely).

Personal self-efficacy is assessed via a six-item scale adapted from Romppel et al. (2013) (e.g., "It is easy for me to stick to my aims and accomplish my goals"). 
Personal self-control is assessed via a three-item scale adapted from Greenaway et al. (2015) (e.g., "I feel in control of my life").

Relationship with service-provider is assessed via an eight-item scale. Five items are adapted from Williams et al. (2015) (e.g., "My Link Worker helps me to feel supported by other people") and one is adapted from Bjerregaard et al. (2015) ("I am willing to do as much as possible with my Link Worker to help achieve my goals").

Activity group attendance is measured for service-users who indicate that they have joined/reconnected with an activity group (e.g., gardening group, craft group) through the pathway. Service-users are asked to indicate how many sessions were available to them to attend in the last 3 months, and of these sessions, how many they actually attended.

A number of other variables are also assessed for service-users who indicate that they have joined/reconnected with an activity group. First, activity group identification is measured with Doosje et al.'s (1995) four-item identification scale (e.g., "I see myself as a member of my activity group").

Second, activity group support is measured with four-item scale adapted from Haslam et al. (2005) (e.g., "I get the emotional support I need from other people in my activity group").

Third, collective efficacy is measured with five-item scale adapted from Reicher and Haslam (2006) (e.g., "My activity group is confident we could deal with unexpected events").

All service-users answer the following final items. Community identification is assessed with five-item scale adapted from McNamara et al. (2013) (e.g., "I see myself as a member of my local community"). Service-users are asked to define 'local community' in any way that is meaningful to them. 
Wellbeing is measured with five-item scale (Heun et al., 2001). Service-users are asked to indicate how often they have experienced each state (e.g., "I have felt cheerful and in good spirits") during the past two weeks, using a 0-5 scale (at no time-all of the time).

\section{(TABLE 1)}

\section{Analysis}

$\underline{\text { Statistical Package for the Social Sciences (SPSS), Mplus (Muthén and Muthén, 2017) }}$ and MLwiN (Charlton et al., 2017) software will be used to conduct the analyses. Demographic information will be described using frequencies and percentages for categorical variables and means (and standard deviations-SDs) or medians (and inter-quartile rangesIQRs). Scores for standardised scales will be described by means (and SDs) or medians (and IQRs), depending on the shape of their distributions.

$\underline{\text { Associations }}$ between predictors and outcomes will be tested using regression. Direct and indirect effects can then be measured through mediation models. Mediation analyses will be conducted using the technique described by Sitko et al. (2014). Differences in health across the time-points will be assessed using Analyses of Covariance to identify significant differences in health whilst controlling for demographic variables. In order to assess associations between predictors and outcomes across time-points, multilevel modelling (MLM) will be used. MLM can simultaneously assess between-subject and within-subject effects between predictors and outcomes using techniques described by Enders and Tofighi (2007). This technique is robust against missing data (Hill and Goldstein, 1998) and does not assume independence between variables (Dierdorff and Ellington, 2012). Finally, causal models will be assessed using cross-lagged panel analysis (Segil and Little, 2012). This will allow covariate effects, reciprocal effects, and causal direction to be assessed. Causal diagrams will be used to identify confounders that have an effect on mediators. In addition, our analyses can examine how individual-level variables and relational variables predict 
perseverance across the pathway. Multilevel regression models will be used to explore the association between predictors and outcomes at baseline and at follow up after 3 months and 6 months, which will enable us to test between-participant and within-participant main effects, interaction effects between factors, covariate effects, and interaction effects between covariates and between participant factors. Causal diagrams will be used to identify confounders that have an effect on mediators. Tests of interaction will also be carried out between factors and follow up times to investigate whether associations change across time.

Correlations and variance inflation factor (VIF) values will be examined in order to test for multicollinearity, and transformations will be applied if data are not approximately normally distributed. Data will be checked to determine whether it is reasonable to assume missingness at random. If appropriate, full information maximum likelihood estimation (FIML) will be used to handle missing data at baseline and follow-ups.

\section{Economic Analysis}

Evidence of economic benefits over time will be obtained if we see a minimal important difference (MID) in the EQ-5D utility index, which is defined as an EQ-5D reduction of at least 0.074 compared to baseline (Walters and Brazier, 2005). A variable will be created at Waves 2, 3, 4 and 5 which will indicate whether or not the service-user has achieved MID at each time-point. A distribution-based approach using standard error of measurement and Cohen's measurement of standardised effect size (Cohen, 1988) will also be used to calculate differences in EQ-5D across the time-points (see Kazis et al., 1989).

Costs for each resource will be derived from the Personal Social Services Research Unit's Annual Survey of Unit Costs of Health and Social Care (Curtis and Burns, 2016) and average costs per service-user will be estimated by dividing total costs by total number of service-users at the intervention's outset. Additionally, generalised linear models will be used to quantify the association between outcome variables, resource use, and healthcare costs. 


\section{Study 2: Interview Study}

The aim of Study 2 is to use semi-structured interviews to gain deeper insight into the lived experiences of those giving and receiving pathway support, including an exploration of aspects that they feel enhance and/or hinder the initiative.

\section{Identification and Recruitment}

Service-users. After the initial survey data have been received by the research team, at least 80 service-users will be selected from the data-file. These service-users will be selected based on their survey responses, with the aim of obtaining as varied a sample as possible (e.g., both sexes, a range of ages and socio-economic statuses, and a variety of scores on the wellbeing measures). The list of selected service-users will be sent to pathway staff, who will post Participant Information Sheets to these individuals. Interested individuals will be asked to telephone the research team in order to arrange an interview. All interested service-users will be interviewed. Assuming a 25\% response rate, we intend to interview around 20 service-users (see Crouch and McKenzie, 2006, who propose a sample size of fewer than 20 in order to facilitate close association with participants). Additionally, interviewing more than 12 service-users will maximise the likelihood of all potential themes within the interviews being captured (Guest et al., 2006).

Service-Providers. Service-providers involved in pathway delivery (e.g. GPs, pathway staff third-sector community group organisers) will be sent Participant Information Sheets via their managers. The sheet will invite them to telephone the research team in order to arrange an interview. A minimum of 20 service-providers will be interviewed.

\section{Interview Schedule}

Each interview is expected to last around one hour and will be audio-recorded. The service-user interview schedule includes questions about the way in which they were recruited into the initiative, their level of engagement with any community activities with 
which they are involved, as well as the perceived impact of these (and other) group memberships on their health, confidence, and quality of life. The service-provider interview questions differ depending upon the specific type of service-provider being interviewed. For community group organisers, questions will explore their perceptions of the organisation for which they work, and the benefits of the organisation for its members. They will also be asked about the extent to which participating in their organisation allows individuals to take ownership of group activities and contribute to long-term sustainability of the organisations. For referrers (e.g., GPs), questions will focus on their core role of profiling the needs of individuals and accurately matching them to referral options. They will also be asked about their perceptions and beliefs concerning SP and their experience of its benefits to their patients. For HCs and LWs, questions will concern their experiences of successes in facilitating 'activation' and 'engagement' among service-users, as well as their observations of attrition and disengagement from the initiative. Together, these interviews will provide rich data exploring the personal experiences of those involved in the pathway, as well as shedding light on the initiative's dynamics.

Consent will be obtained by the research assistant in-person before each interview. Participants will be debriefed and directed to relevant support services if they have been affected by anything discussed in the interview.

\section{Analysis}

The interviews will be transcribed verbatim and the transcripts analysed using theoretically-driven thematic analysis, as outlined by Braun and Clarke (2013). This will provide a systematic overview of the ways in which both sets of participants understand and experience SP, and allow comparison of where these understandings converge and diverge.

\section{Ethical Considerations}


Ethical approval for all aspects of this project have been obtained from our institution's Research Ethics Committee (code 2018/58) and NHS England Research Ethics Committee (IRAS code: 235506).

Pathway staff involved in data collection are trained to ask potentially distressing questions with sensitivity and awareness. Their training includes guidance on clinical assessment, assessing individuals' capacity to consent, effective communication with vulnerable populations, and knowledge of NHS policies on working with vulnerable populations, as well as ethical data collection/storage. All pathway staff are employed by the local NHS Trust, and thus benefit from professional indemnity insurance, and are regulated under the complaints mechanisms of the Trust. Pathway staff are also provided with regular individual and group supervision.

It is unlikely that the questions asked in this project will raise strong emotional responses from the participants, but if they do, the pathway staff are trained to address such concerns professionally. Participants will be offered one or more of the following options as appropriate: to not answer any questions they find distressing, to take a break from the survey/interview, to terminate the survey/interview, or to talk to a professional counsellor. Participants will be reminded that withdrawing from the study (or choosing not to participate) will not affect their care.

\section{Confidentiality}

Regarding Study 1, all clinical assessment and research data will be stored on the NHS SystmOne database. Data will be pseudo-anonymised at the point of collection and collated into a data-file held by the pathway staff and only pseudo-anonymised data will be passed to the research team for analysis. All pseudo-anonymised data-files to which the researches have access will be stored on password-protected university computers. 
Regarding Study 2, any identifying information collected during interviews will be kept separate from the interview data and in a locked cupboard in the Principal Investigator's office. Interviewees will be given pseudonyms during transcription, and any names of people or places (including the names of GP surgeries) or other identifying information will be redacted.

\section{Discussion}

This project will be the first to use the Social Cure approach (Jetten et al., 2012) to evaluate an NHS SP initiative. The work is intended to evaluate the impact of this pathway on service-users' health, wellbeing, and service use, as well as the Social Cure processes we believe will drive these benefits. The wide range of self-report and standardised measures along with in-depth interview data will create a rich data corpus, enabling us to explore both subjective and objective intervention aspects.

Specifically, we expect to observe a relationship between isolation, loneliness, and poor wellbeing at the start of the intervention, highlighting service-user needs that cannot be met by primary care services. Through the longitudinal assessment we expect to find improvements in wellbeing and reduced loneliness amongst those engaging with the intervention. We expect the improvement to be mediated, in part, by increased group identification, support, and efficacy, and to become stronger as the user/provider relationship strengthens. In turn, these changes will contribute to reduced service usage at follow-up. In this way we expect to identify the 'active ingredients' within the pathway. We also expect to observe an economic impact of the pathway and intend to provide an insight into the specific elements of service-users' treatment that lead to this financial outcome. Finally, the qualitative study will allow us to explore service-users' own experiences of the pathway, how these match/differ from that of service-providers, and what the consequences of divergent understandings might be for service-user outcomes. The generalisations of these findings will 
necessarily be limited by the specificities of the delivery model adopted, the particularities of the local community and social resources available to the pathway, as well as the serviceusers' needs. However, our triangulation of methods, longitudinal design, and patient-centred approach will shed necessary light on how SP has its effects; knowledge which can be used to inform the development of this and other models.

At a time when national health services are struggling to cope with increased demand and long-term health issues exacerbated by loneliness, social engagement can provide a costeffective and sustainable solution. The increased sense of control, esteem, and social support that results from identification with social groups is evidenced in the Social Cure perspective (Jetten et al., 2012) in terms of resultant improvements in wellbeing, but also in terms of continued engagement, thereby creating a sustainable resource for the individual and their community. The present research proposes an efficient model for understanding and applying $\underline{\text { the Social Cure to a new context through developing collaboration between formal service- }}$ providers and community organisations.

\section{Declarations}

\section{Conflicting Interests}

None of the research team members report a conflict of interest.

\section{Funding}

The project is funded by ImROC, an NHS-based organisation. There is no grant number for this funding. 


\section{References}

Bjerregaard K, Haslam SA, Morton T, Ryan MK (2015) Social and relational identification as determinants of care workers' motivation and well-being. Frontiers in Psychology 6:1460.

Brandling J and House W (2009) Social prescribing in general practice: Adding meaning to medicine. British Journal of General Practice 59(563): 454-456.

Braun V and Clarke, V (2013) Successful qualitative research: A practical guide for beginners. London: Sage.

Cawston P (2011) Social prescribing in very deprived areas. British Journal of General Practice 61(586): 350-350.

Charlton C, Rasbash J, Browne WJ, Healy M, Cameron B (2017) MLwiN Version 3.00. $\underline{\text { Centre for Multilevel Modelling, University of Bristol. }}$

Cohen J (1988) Statistical power analysis for the behavioural sciences. Mahwah, NJ: Lawrence Erlbaum.

Crouch M and McKenzie H (2006) The logic of small samples in interview-based qualitative research. Social Science Information 45(4): 483-499.

Curtis L and Burns A (2016) Unit costs of health and social care. Available from: http://www.pssru.ac.uk/project-pages/unit-costs/unit-costs-2016/ (accessed 5 April 2018).

Dayson C and Bashir N (2014) The social and economic impact of the Rotherham Social Prescribing Pilot: Main evaluation report. Centre for Regional Economic and Social Research: Sheffield Hallam University. Available at: http://shura.shu.ac.uk/18961/ (accessed 28 February 2018). 
Dierdorff EC and Ellington JK (2012) Members matter in team training: Multilevel and longitudinal relationships between goal orientation, self- regulation, and team outcomes. Personnel Psychology 65(3): 661-703.

Doosje B, Ellemers N and Spears R (1995) Perceived intragroup variability as a function of group status and identification. Journal of Experimental Social Psychology 31: 410436.

Enders C and Tofighi D (2007) Centering predictor variables in cross-sectional multilevel models: a new look at an old issue. Psychological Methods 12(2): 121.

EuroQol, Group (1990). EuroQol--a new facility for the measurement of health-related quality of life. Health Policy (Amsterdam, Netherlands) 16(3): 199-208.

Grant C, Goodenough T, Harvey I and Hine C (2000) A randomized controlled trial and economic evaluation of a referrals facilitator between primary care and the voluntary sector. British Medical Journal 320: 419-23.

Greenaway KH, Haslam SA, Cruwys T, Branscombe NR, Ysseldyk R and Heldreth C (2015) From "we" to "me": Group identification enhances perceived personal control with consequences for health and well-being. Journal of Personality and Social Psychology 109(1): 53-74.

Guest G, Bunce A and Johnson L (2006) How many interviews are enough? An experiment with data saturation and variability. Field Methods 18(1): 59-82.

Haslam C, Cruwys T, Haslam SA, Dingle G, Chang MXL (2016) Groups4Health: Evidence that a social-identity intervention that builds and strengthens social group membership improves mental health. Journal of Affective Disorders 194:188-195.

Haslam, C, Jetten J, Cruwys T, Dingle G, Haslam SA (2018) The new psychology of health: Unlocking the social cure. London: Routledge. 
Haslam SA, O’Brien A, Jetten J, Vormedal K and Penna S (2005) Taking the strain: Social identity, social support, and the experience of stress. British Journal of Social Psychology 44: 355-370.

Hawkley LC and Cacioppo JT (2010) Loneliness matters: A theoretical and empirical review of consequences and mechanisms. Annals of Behavioral Medicine 40(2): 218-227.

Hays RD and DiMatteo MR (1987) A short-form measure of loneliness. Journal of Personality Assessment 51(1): 69-81.

Heun R, Bonsignore M, Barkow K, Jessen F (2001) Validity of the five-item WHO WellBeing Index (WHO-5) in an elderly population. European Archives of Psychiatry and Clinical Neuroscience. 251:27-31.

Hibbard JH, Stockard J, Mahoney ER, Tusler M (2005) Development and testing of a short form of the patient activation measure. Health Services Research 40(6): 1918-1930. Hill PW and Goldstein H (1998) Multilevel modeling of educational data with crossclassification and missing identification for units. Journal of Educational and Behavioral Statistics 23(2): 117-128.

Iyer A, Jetten J, Tsivrikos D, Postmes T, Haslam SA (2009) The more (and the more compatible) the merrier: Multiple group memberships and identity compatibility as predictors of adjustment after life transitions. British Journal of Social Psychology 48(4): 707-733.

Jetten J, Haslam C and Haslam SA (2012) The social cure: Identity, health and well-being. Hove: Psychology Press.

Jick TD (1979) Mixing qualitative and quantitative methods: Triangulation in action. Administrative Science Quarterly 24(4): 602-611. 
Local Government Association (2017) Budget 2017: LGA responds to social care funding. Available at: https://www.local.gov.uk/about/news/budget-2017-lga-responds-socialcare-funding (accessed 4 March 2018).

Kazis LE, Anderson JJ and Meenan RF (1989) Effect sizes for interpreting changes in health status. Medical Care 27(3): 178-189.

$\underline{\text { Kellezi B, Baines DL, Coupland C, Beckett K, Barnes J, Sleney J, Christie N, Kendrick D }}$ (2016) The impact of injuries on health service resource use and costs in primary and secondary care in the English NHS. Journal of Public Health, 38(4), e464-e471.

Kellezi B, Reicher S, Cassidy C (2009) Surviving the Kosovo conflict: A Study of social identity, appraisal of extreme events, and mental well- being. Applied Psychology: An International Review 58(1):59-83.

$\underline{\text { Kimberlee R (2015) What is social prescribing? Advances in Social Sciences Research }}$ Journal, 2(1).

Kimberlee R (2016) Gloucestershire Clinical Commissioning Group's Social Prescribing Service: Evaluation report. 2016. Available at: http://eprints.uwe.ac.uk/30293/3/Report\%25406.pdf (accessed 5 March 2018).

Kimberlee R, Ward R, Jones M and Powell J (2014) Measuring the economic impact of Wellspring Healthy Living Centre's Social Prescribing Wellbeing Programme for low level mental health issues encountered by GP services. Available at: http://www.wellspringhlc.org.uk/reports/POV_Final_Report_March_2014.pdf (accessed 3 March 2018).

King's Fund (2017) What is social prescribing? Available at: https://www.kingsfund.org.uk/publications/social-prescribing (accessed 28 February 2018). 
McNamara N, Stevenson C and Muldoon OT (2013) Community identity as resource and context: A mixed method investigation of coping and collective action in a disadvantaged community. European Journal of Social Psychology 43(5): 393-403.

Muthén LK and Muthén BO (2017) Mplus user's guide. Sixth edition. Los Angeles, CA: Muthén \& Muthén.

Office for National Statistics (2017). National Population Projections: 2016-based statistical bulletin. Available from:

https://www.ons.gov.uk/peoplepopulationandcommunity/populationandmigration/pop ulationprojections/bulletins/nationalpopulationprojections/2016basedstatisticalbulletin (accessed 28 February 2018).

Reicher S and Haslam SA (2006) Rethinking the psychology of tyranny: The BBC prison study. The British Journal of Social Psychology 45: 1-40.

Romppel M, Herrmann-Lingen C, Wachter R, Edelmann F, Düngen HD, Pieske B and Grande G. A (2013) Short form of the General Self-Efficacy Scale (GSE-6): Development, psychometric properties and validity in an intercultural non-clinical sample and a sample of patients at risk for heart failure. GMS Psycho-Social-Medicine 10: Doc01.

Sani F, Madhok V, Norbury M, Dugard P, Wakefield JRH (2015) Greater number of group identifications is associated with healthier behaviour: Evidence from a Scottish community sample. British Journal of Health Psychology 20(3): 466-481.

Selig JP and Little TD (2012) Autoregressive and cross-lagged panel analysis for longitudinal data. In B Laursen, TD Little and NA Card (Eds.) Handbook of developmental research methods (pp. 265-278). New York: Guilford Press.

$\underline{\text { Sitko K, Bentall RP, Shevlin M, O’Sullivan N and Sellwood W (2014) Associations between }}$ $\underline{\text { specific psychotic symptoms and specific childhood adversities are mediated by }}$ 
attachment styles: an analysis of the National Comorbidity Survey. Psychiatry Research 217(3): 202-209.

South J, Higgins TJ, Woodall J and White SM. (2008) Can social prescribing provide the missing link? Primary Health Care Research \& Development 9(4): 310-318.

Stickley T and Hui A (2012) Social prescribing through arts on prescription in a UK city: Referrers' perspectives. Public Health 126(7): 580-586.

Thomson LJ, Camic PM and Chatterjee HJ (2015) Social prescribing: A review of community referral schemes. London: University College London.

Tajfel H and Turner JC (1986) The social identity theory of intergroup behavior. In: Worchel S and Austin LW (eds) Psychology of intergroup relations (2nd ed). Chicago, It: Nelson-Hall; pp. 7-24.

Turner JC, Hogg MA, Oakes, PJ, Reicher SD, and Wetherell MS (1987) Rediscovering the social group. Oxford, UK: Blackwell.

Wakefield JRH, Bickley S and Sani F (2013) The effects of identification with a support group on the mental health of people with multiple sclerosis. Journal of Psychosomatic Research 74(5): 420-426.

Walters SJ, Brazier JE. (2005). Comparison of the minimally important difference for two health state utility measures: EQ-5D and SF-6D. Quality of Life Research 14(6):1523-1532.

Williams J, Leamy M, Bird V, Le Boutillier C, Norton S, Pesola F and Slade M (2015) Development and evaluation of the INSPIRE measure of staff support for personal recovery. Social Psychiatry and Psychiatric Epidemiology 50: 777-786. 
Table 1. Survey measures included at each time-point

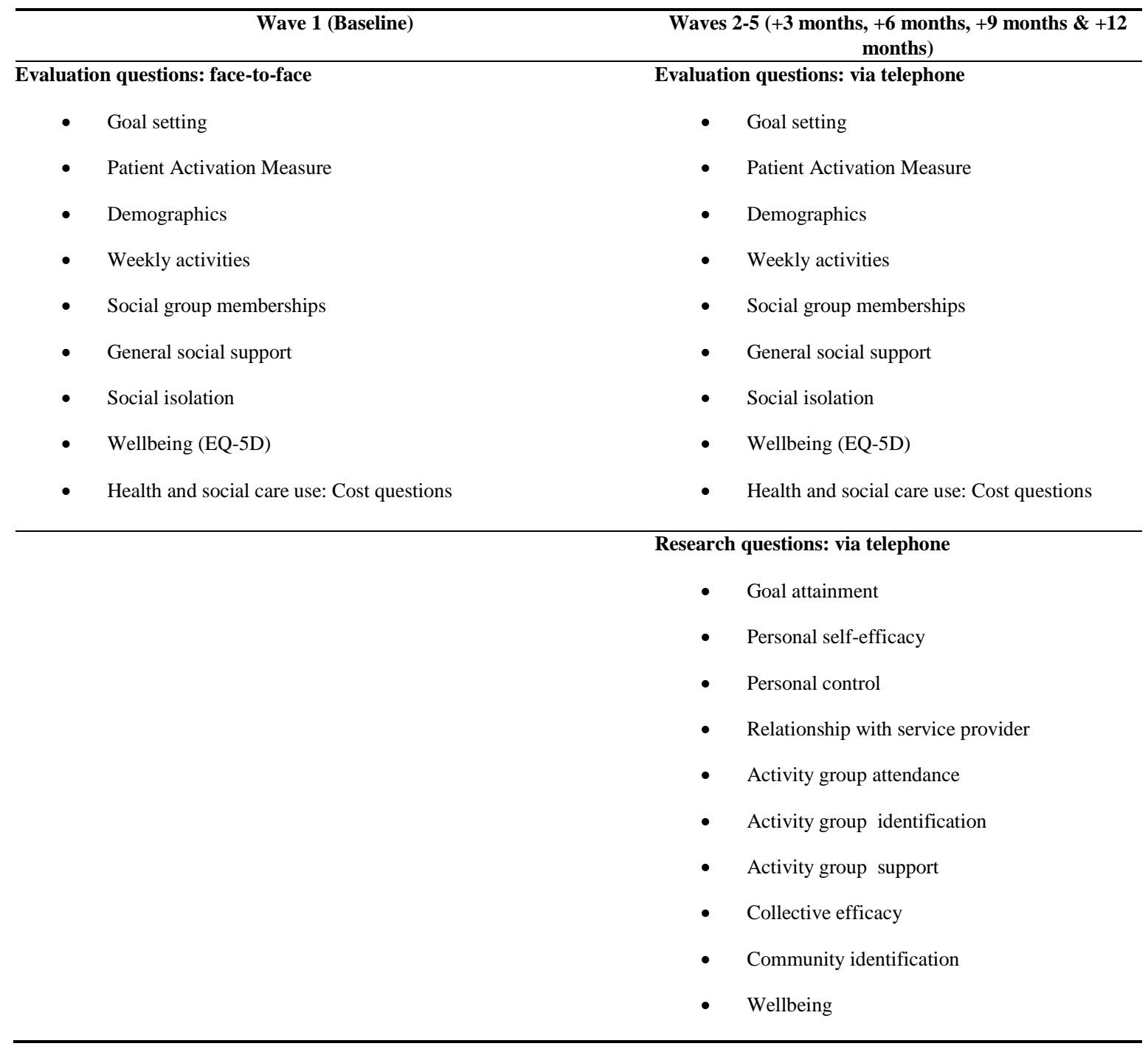

Note: Additional consent is required from service-users to take part in the research survey 